bupaR: Enabling reproducible business process analysis

Non Peer-reviewed author version

JANSSENSWILLEN, Gert; DEPAIRE, Benoit; SWENNEN, Marijke; JANS, Mieke \& VANHOOF, Koen (2018) bupaR: Enabling reproducible business process analysis.

In: Knowledge-based systems, 163, p. 927-930.

DOI: 10.1016/j.knosys.2018.10.018

Handle: http://hdl.handle.net/1942/27485 


\title{
bupaR: Enabling Reproducible Business Process Analysis
}

\author{
Gert Janssenswillen ${ }^{\mathrm{a}, \mathrm{b}}$, Benoît Depaire ${ }^{\mathrm{a}}$, Marijke Swennen ${ }^{\mathrm{a}}$, Mieke Jans ${ }^{\mathrm{a}}$, \\ Koen Vanhoof ${ }^{\mathrm{a}}$ \\ ${ }^{a}$ Faculty of Business Economics \\ UHasselt - Hasselt University \\ Martelarenlaan 42 3500 Hasselt, Belgium \\ ${ }^{b}$ Flemish Research Foundation \\ Egmontstraat 61000 Brussels, Belgium
}

\begin{abstract}
Over the last decades, the field of process mining has emerged as a response to a growing amount of event data being recorded in the context of business processes. Concurrently with the increasing amount of literature produced in this field, a set of tools has been developed to implement the various algorithms and provide them to end users. However, the majority of tools does not provide the possibility of creating workflows which can be reused at a later point in time to reproduce the results, and most tools are not easily customizable. This paper introduces bupaR, an integrated collection of Rpackages which creates a framework for reproducible process analysis in $\mathrm{R}$ and supports different steps of a process analysis project, from data extraction to data analysis. It is an extensible framework of several R-packages to analyse process data, each with their specific purpose and set of tools.
\end{abstract}

Keywords: event data, process analysis, R, bupaR, edeaR, eventdataR, processmapR, processmonitR, xesreadR

\section{Introduction}

Over the last decades, the field of process mining has arisen as a response 3 to a growing amount of event data being recorded in the context of business 4 processes. Pioneering works considered the discovery of process models from 5 event data $[1,2,3]$, known as process discovery. Insights from the analyses 6 soon proofed to be highly beneficial for companies to improve performance 
7

and quality, which has caused an enormous volume of process analytics research, encompassing a wide range of techniques and algorithms to analyse event data [4].

Concurrently with the increasing amount of literature produced in this field, a set of tools has been developed to implement the various algorithms and provide them to end users. The tools that were developed are both academic and commercial in nature, and are diverse concerning their customizability, implementation platform or architecture, and the techniques they support. However, the existing tools have several drawbacks. Firstly, the majority of tools do not provide the possibility of creating workflows which can be reused at a later point in time to reproduce the results. Secondly, since they aim to support any possible process, most tools are not (easily) customizable, by adequately taking into account custom data attributes, or by allowing visualization to be customized according to the process context or sector. Finally, the majority of tools are stand-alone programs, solely supporting process mining techniques, without an interface to general-purpose data mining, visualization or statistical tools.

This paper introduces bupaR, a collection of R-packages which provide a framework for reproducible process analysis in $\mathrm{R}$. The packages implement a class for event data in $\mathrm{R}$, together with a set of generics and methods to handle it. By providing support for process analysis in $\mathrm{R}$, it is the first tool to analyse processes using reusable scripts as well as to combine scripts, meta-data and interpretation of the results with Rmarkdown documents. The framework currently contains techniques for exploratory and descriptive event data analysis, for visualizing process data with process maps, and for creating real-time process monitoring dashboards, among other things.

\section{Problems and Background}

Process mining originated at the end of the $20^{\text {th }}$ century with the development of algorithms trying to learn models from event data [1,2,3]. Over the years, many more advanced algorithms for process discovery were developed, such as the heuristics miner [5], ILP miner [6] and inductive miner [7]. Next to process discovery, conformance checking emerged as another important research track within process mining [8]. The latter is focused on the relation between event data on the one hand and the process model on the other hand. It aims at finding inconsistencies between the two and furthermore 


\begin{tabular}{|c|c|c|}
\hline Package & Version & Functionality \\
\hline bupaR [13] & 0.4 .0 & $\begin{array}{l}\text { Creation and handling of event log objects } \\
\text { and basic preprocessing tasks }\end{array}$ \\
\hline edeaR $[14]$ & 0.8 .0 & Calculate descriptive process metrics \\
\hline eventdataR $[15]$ & 0.2 .0 & Contains example event data \\
\hline xesreadR $[16]$ & 0.2 .2 & Read and write .XES-files \\
\hline processmapR [17] & 0.3 .1 & $\begin{array}{l}\text { Draw process map and other process specific } \\
\text { visualization }\end{array}$ \\
\hline processanimateR [18] & 0.1 .1 & Animate process maps \\
\hline petrinetR $[19]$ & 0.1 .0 & Read and handle Petri Nets \\
\hline processmonitR [20] & 0.1 .0 & $\begin{array}{l}\text { Create interactive dashboards for process } \\
\text { analysis }\end{array}$ \\
\hline
\end{tabular}

Table 1: Current packages in the bupaR framework.

\section{assesses the performance of discovery algorithms in their attempt to find a good representation of the process captured with the event data. \\ Although process discovery and conformance checking are still important topics in the process mining domain, it has recently grown much bigger. Currently, attention is given to real-time process analysis [9], blockchain [10], Internet-of-things [11], and predictive process monitoring [12], among others. The focus of bupaR currently is on the sub domain of process analytics, focusing entirely on the analysis of process data, and is less concerned with executable process models. In this sense, it is similar to most commercial process analysis tools.}

\section{Software Architecture and Functionalities}

An overview of the different packages contained by the bupaR framework is given in Table 1. Note that the name bupaR refers to the overall framework as well as to the central package for supporting event data. We will generally use the term to refer to the overall framework, unless we explicitly stated otherwise. In the next paragraphs, the functionalities of each of the packages is discussed in more detail.

bupaR. The bupaR-package [13] is the core package of the framework, implements an S3-objects class for event data. It provides functions to create these objects, as well as support for common transformations. Auxiliary functions to seamlessly change the classifiers of the event data are made available, and 
event log versions of common dplyr [21] functions for data manipulation are implemented, such as filter, group_by and mutate, among others. These functions can be used to preprocess event data. Some specific preprocessing tasks are supported explicitly by specific functions, such as aggregations of activity labels.

edeaR. edeaR [14] stands for Exploratory and Descriptive Event-Data Analyses, and contains a set of process metrics to describe and explore event logs. The process metrics are based on Lean Six Sigma literature [22] and can be analyzed and visualized at different levels of granularity. Additionally, edeaR contains an extensive collection of event data specific filters.

eventdataR. eventdataR [15] is a data-package which provide easy access to event logs for testing and experiments. Currently, both artificial event data, e.g. patients, as well as real-life event data, such as the Sepsis dataset [23]. xesreadR. In order to be compatible with the eXtensible Event Stream IEEE standard [24], the xesreadR package [16] allow to read and write .xes-files.

processmapR. Process data specific visualizations, such as process maps and dotted charts [25], are provided by processmapR [17]. As a result, processmapR is complementary to edeaR for exploring and describing process data, where the latter focuses more on numeric result and processmapR on visualizations.

processanimateR. By extending processmapR, processanimateR [18] allows to easily animate process maps using token replay.

processmonitR. In order to facilitate the creation of dashboards using Shiny [26], processmonitR [20] provides a limited set of process dashboards, focussed on a specific aspect, e.g. performance, resources, etc. These can be used in a permanent, real-time fashion, as well as for interactive data analysis. While still in an experimental phase, the goal is to extend this package to allow for easy building of custom process dashboards. Furthermore, built-in support for online analysis using partial cases and using event streams can be added in the future.

petrinetR. While all the package above are centered around process data, petrinetR [19] is the first package to introduce a notion of process models in R. Currently, the main functionality is to create, read and write Petri Nets, to adjust them, visualize them, but also to perform token replay and parse 
transition sequences. In future, the goal is the link this package with the other packages by means of process discovery and conformance checking.

\section{Comparison with other process analysis tools}

In comparison with existing tools for process analytics, both open-source and commercial, bupaR can be seen is unique as it 1) is easily extensible and combinable with other tools, 2) allows to reproduce workflows, and is 3) interactive, supporting and iterative and dynamic user interaction [27].

One of the most extensive and open-source process mining framework to date is ProM [24]. It contains most of the state-of-the-art techniques which are developed in related literature. It can be extended with java-libraries, although it requires a considerable time investment to do so, as one has to be familiar with the source-code of the central frame-work. Furthermore, its setup, with a click-and-select user interface, makes it hard to make your analysis reproducible. In order to enable reproducible workflows, RapidProM [28], an extension to RapidMiner, was developed. RapidProM allows the execution of the most widely used ProM-plugins within RapidMiner. As a result, RapidProM supports reproducible process analysis workflows, using the RapidMiner GUI of dropping and connect operators, and provides an interface with all the other data analysis techniques available in RapidMiner. Also the interactiveness of RapidProm is rather low, as assembling a workflow typically requires a clear goal decided upon beforehand, and altering workflows can be cumbersome. Other commercial tooling score higher on interactiveness, especially due to the use of interactive graphical visualizations (e.g. Disco ${ }^{1}$, Celonis $^{2}$ ). However, reproducibility and extensibility is generally very low.

Some support for event data in its broadest sense is already available in the form of several R packages. For instance, the events package [29] uses the KEDS (Kansas Event Data System) format [30]. This format is targeted at political event data, and typically extracted from news reports. In addition, eventstudies [31] regards event data as a dataset with two columns, name and when. It thus contains information about when a specific event happened for a certain subject. It is clear that none of these existing packages support the

\footnotetext{
${ }^{1}$ https://fluxicon.com/disco/

${ }^{2}$ https://www.celonis.com/
} 
more complex data structures typical for business process data, nor do they provide the required tools to analyze these.

\section{Applications and Illustrative Examples}

The bupaR framework has been applied in academic works such as [32], project such as the H2020 project $\mathrm{HUMAN}^{3}$, as well as by professionals ${ }^{4}$

Some examples of functionalities are shown in Figure 1, which can be created using the $\mathrm{R}$ statements below. Figure 1a shows a process map, colored according to the processing time of activities. Figure 1b shows a dotted chart, which displays how activities are distributed along the time of day. Figure 1c shows a resource-activity matrix, where one can observe which resources executed which activities. The data used in these examples can be found in the eventdataR package. For more examples, we refer to the documentation and website. ${ }^{5}$

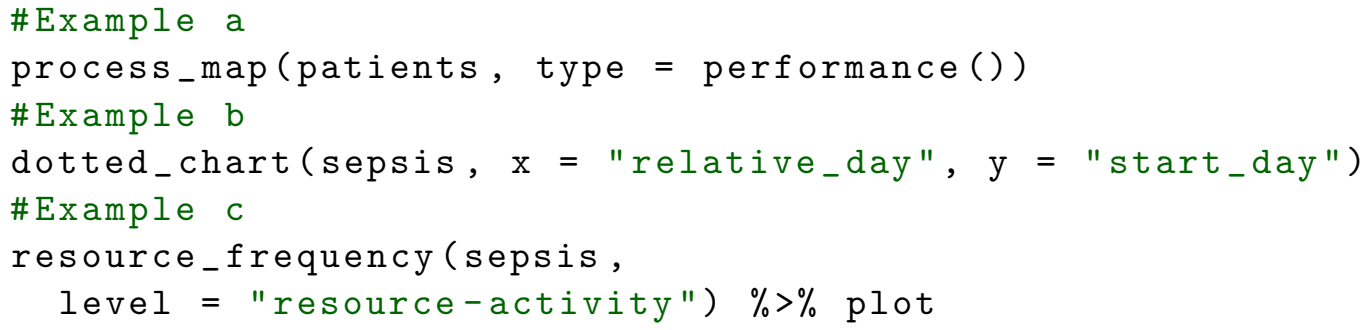

\section{Conclusions}

In this paper, we introduced a collection of R-packages which were designed to support the different analytical stages within process analysis, from the data extraction to the analysis and mining. It is the first effort to support the handling and analysis of process event data in $\mathrm{R}$.

Making process analysis possible in $\mathrm{R}$ will improve the reproducibility of process analyses. Reusable analysis scripts can be combined with the interpretation of the analysis as well as with meta-data. Furthermore, it will

\footnotetext{
${ }^{3}$ http://humanmanufacturing.eu

${ }^{4}$ https://medium.com/@gscheithauer/process-mining-in-10-minutes-with-r1ab28ed74e81

${ }^{5}$ http://bupar.net
} 


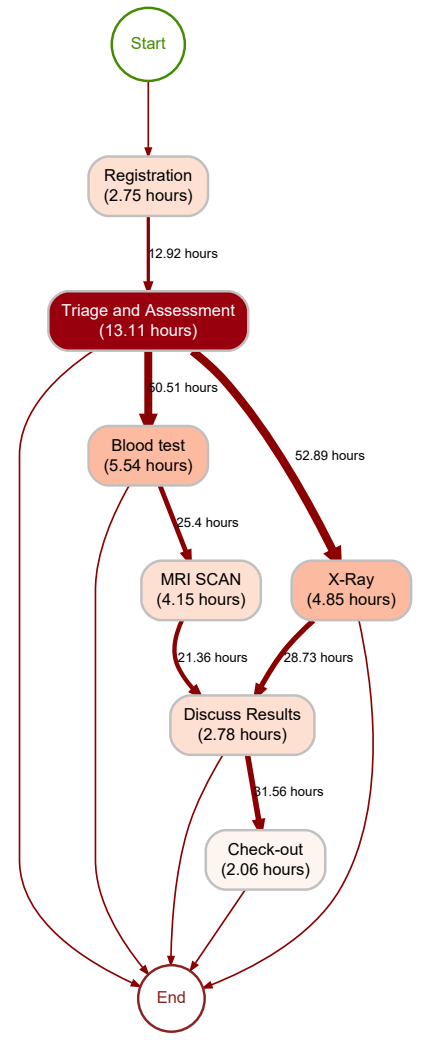

(a)

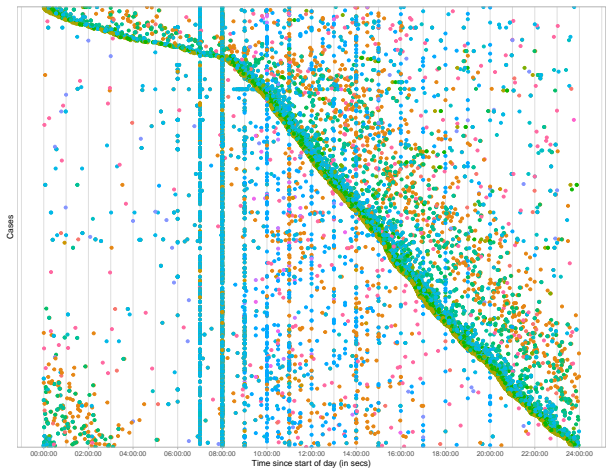

(b)

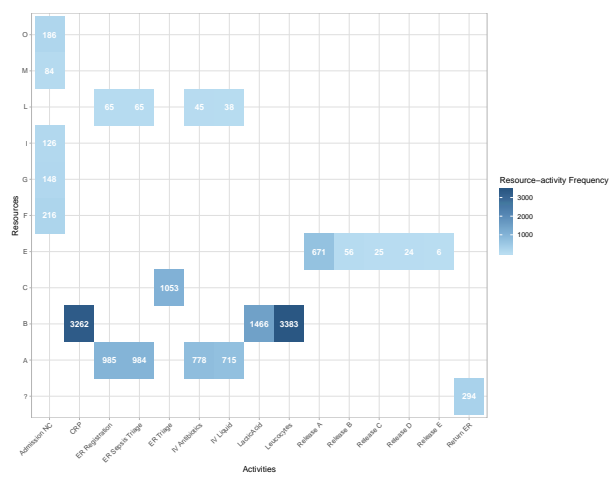

(c)

Figure 1: Examples of process visualizations 
allow process analysts to easily create custom analysis tools, and will enlarge the adoption and publicity of process mining in industry.

Further extensions to the framework are planned for the near future, in order to resolve some important limitations of current functionalities. Foremost, the support for working with executable process models in $\mathrm{R}$, such as Petri Nets and BPMN models should be improved. Subsequently, we believe that providing process discovery algorithms and conformance checking are important next steps, in order to support end-to-end process analysis. The best way to do this, by reimplementing existing approaches, or by creating interfaces with other tools, still has to be decided upon.

\section{References}

[1] J. E. Cook, A. L. Wolf, Automating process discovery through eventdata analysis, in: Software Engineering, 1995. ICSE 1995. 17th International Conference on, IEEE, 1995, pp. 73-73.

[2] R. Agrawal, D. Gunopulos, F. Leymann, Mining process models from workflow logs, in: H. J. Schek, F. Saltor, I. Ramos, G. Alonso (Eds.), Advances in Database Technology - EDBT '98, Vol. 1377, SpringerVerlag Berlin Heidelberg, 1998, pp. 467-483.

[3] A. Datta, Automating the discovery of as-is business process models: Probabilistic and algorithmic approaches, Information Systems Research 9 (3) (1998) 275-301.

[4] W. van der Aalst, Process mining: discovery, conformance and enhancement of business processes, Springer, Heidelberg, 2011.

[5] A. Weijters, J. Ribeiro, Flexible Heuristics Miner (FHM), in: 2011 IEEE Symposium on Computational Intelligence and Data Mining (CIDM), 2011, pp. 310-317.

[6] J. M. E. Van der Werf, B. F. van Dongen, C. A. Hurkens, A. Serebrenik, Process discovery using integer linear programming, in: Applications and Theory of Petri Nets, Springer, 2008, pp. 368-387.

[7] S. J. J. Leemans, D. Fahland, W. M. P. van der Aalst, Discovering blockstructured process models from event logs-a constructive approach, in: Application and Theory of Petri Nets and Concurrency, Springer, 2013, pp. 311-329. 
[8] A. Rozinat, W. M. P. van der Aalst, Conformance checking of processes based on monitoring real behavior, Information Systems 33 (1) (2008) 64-95.

[9] F. M. Maggi, A. Burattin, M. Cimitile, A. Sperduti, Online Process Discovery to Detect Concept Drifts in LTL-Based Declarative Process Models, in: On the Move to Meaningful Internet Systems: OTM 2013 Conferences, Lecture Notes in Computer Science, Springer, Berlin, Heidelberg, 2013, pp. 94-111.

[10] I. Weber, X. Xu, R. Riveret, G. Governatori, A. Ponomarev, J. Mendling, Untrusted business process monitoring and execution using blockchain, in: International Conference on Business Process Management, Springer, 2016, pp. 329-347.

[11] C. Janiesch, A. Koschmider, M. Mecella, B. Weber, A. Burattin, C. Di Ciccio, A. Gal, U. Kannengiesser, F. Mannhardt, J. Mendling, A. Oberweis, M. Reichert, S. Rinderle-Ma, W. Song, J. Su, V. Torres, M. Weidlich, M. Weske, L. Zhang, The Internet-of-Things Meets Business Process Management: Mutual Benefits and Challenges, arXiv:1709.03628 [cs]ArXiv: 1709.03628. URL http://arxiv.org/abs/1709.03628

[12] F. Folino, M. Guarascio, L. Pontieri, Mining predictive process models out of low-level multidimensional logs, in: International conference on advanced information systems engineering, Springer, 2014, pp. 533-547.

[13] G. Janssenswillen, bupaR: Business Process Analytics in R, r package version 0.3.0 (2017).

[14] G. Janssenswillen, M. Swennen, edeaR: Exploratory and Descriptive Event-Based Data Analysis, r package version 0.7.1 (2017).

[15] G. Janssenswillen, eventdataR: Event Data Repository, r package version 0.1.1 (2017).

[16] G. Janssenswillen, B. Depaire, xesreadR: Read and Write XES Files, r package version 0.2.1 (2017).

[17] G. Janssenswillen, processmapR: Construct Process Maps Using Event Data, r package version 0.2.0 (2017). 
[18] F. Mannhardt, processanimateR: Process Map Animation (2018).

[19] G. Janssenswillen, petrinetR: Building, Visualizing, Exporting and Replaying Petri Nets, r package version 0.1.0 (2016).

[20] G. Janssenswillen, processmonitR: Building Process Monitoring Dashboards, r package version 0.1 .0 (2017).

URL https : //CRAN.R-project.org/package=processmonitR

[21] H. Wickham, R. Franois, L. Henry, K. Mller, dplyr: A Grammar of Data Manipulation, R package version 0.7 .5 (2018).

URL https : //CRAN.R-project.org/package=dplyr

[22] M. Swennen, G. Janssenswillen, M. J. Jans, B. Depaire, K. Vanhoof, Capturing Process Behavior with Log-Based Process Metrics, in: Proceedings of the 5th International Symposium on Data-driven Process Discovery and Analysis (SIMPDA), Vienna, 2015.

[23] F. Mannhardt, Sepsis Cases - Event Log, dOI: 10.4121/uuid:915d2bfb7e84-49ad-a286-dc35f063a460 (2016).

[24] H. M. W. Verbeek, J. C. A. M. Buijs, B. F. Van Dongen, W. M. P. Van Der Aalst, Xes, xesame, and prom 6, in: P. Soffer, E. Proper (Eds.), Information Systems Evolution, Springer, 2011, pp. 60-75.

[25] M. Song, W. M. van der Aalst, Supporting process mining by showing events at a glance, in: Proceedings of the 17th Annual Workshop on Information Technologies and Systems (WITS), 2007, pp. 139-145.

[26] W. Chang, J. Cheng, J. Allaire, Y. Xie, J. McPherson, shiny: Web Application Framework for R, r package version 1.1.0 (2018).

URL https : //CRAN.R-project.org/package=shiny

[27] L. Anselin, Interactive techniques and exploratory spatial data analysis, Geographical Information Systems: principles, techniques, management and applications 1 (1999) 251-264.

[28] W. M. P. van der Aalst, A. Bolt, S. J. van Zelst, RapidProM: Mine Your Processes and Not Just Your Data, arXiv:1703.03740 [cs]ArXiv: 1703.03740.

URL http://arxiv.org/abs/1703.03740 
[29] W. Lowe, events: Store and manipulate event data, $r$ package version 0.5 (2012).

[30] P. A. Schrodt, S. G. Davis, J. L. Weddle, Political science: KEDSa program for the machine coding of event data, Social Science Computer Review 12 (4) (1994) 561-587.

[31] C. Anand, V. Balasubramaniam, V. Bahure, A. Shah, eventstudies: An $\mathrm{R}$ package for conducting event studies and a platform for methodological research on event studies. (2014).

[32] D. Etinger, T. Orehovački, S. Babić, Applying process mining techniques to learning management systems for educational process model discovery and analysis, in: International Conference on Intelligent Human Systems Integration, Springer, 2018, pp. 420-425. 


\begin{tabular}{|c|c|c|}
\hline Nr. & Code metadata description & Please fill in this column \\
\hline C1 & Current code version & 0.3 .2 \\
\hline $\mathrm{C} 2$ & $\begin{array}{l}\text { Permanent link to code/repository } \\
\text { used of this code version }\end{array}$ & https://github.com/cran/bupaR \\
\hline C3 & Legal Code License & MIT-License \\
\hline $\mathrm{C} 4$ & Code versioning system used & git \\
\hline $\mathrm{C} 5$ & $\begin{array}{l}\text { Software code languages, tools, and } \\
\text { services used }\end{array}$ & $\mathrm{R}$ \\
\hline C6 & $\begin{array}{l}\text { Compilation requirements, operat- } \\
\text { ing environments \& dependencies }\end{array}$ & \\
\hline $\mathrm{C} 7$ & $\begin{array}{l}\text { If available Link to developer docu- } \\
\text { mentation/manual }\end{array}$ & http : //www.bupar.net \\
\hline $\mathrm{C} 8$ & Support email for questions & gert.janssenswillen@uhasselt.be \\
\hline
\end{tabular}

Table 2: Code metadata 